\title{
BUNDLE GERBES AND SURFACE HOLONOMY
}

\author{
Jürgen Fuchs ${ }^{1}$, Thomas Nikolaus ${ }^{2}$, Christoph Schweigert ${ }^{2}$, Konrad Waldorf ${ }^{3}$ \\ ${ }^{1}$ Teoretisk fysik \\ Karlstads Universitet \\ Universitetsgatan 21 \\ S- 65188 Karlstad \\ 2 Organisationseinheit Mathematik \\ Bereich Algebra und Zahlentheorie \\ Universität Hamburg \\ Bundesstraße 55 \\ D - 20146 Hamburg \\ ${ }^{3}$ Department of Mathematics \\ University of California, Berkeley \\ 970 Evans Hall \#3840 \\ Berkeley, CA 94720, USA
}

\begin{abstract}
Hermitian bundle gerbes with connection are geometric objects for which a notion of surface holonomy can be defined for closed oriented surfaces. We systematically introduce bundle gerbes by closing the pre-stack of trivial bundle gerbes under descent.

Inspired by structures arising in a representation theoretic approach to rational conformal field theories, we introduce geometric structure that is appropriate to define surface holonomy in more general situations: Jandl gerbes for unoriented surfaces, D-branes for surfaces with boundaries, and bi-branes for surfaces with defect lines.
\end{abstract}




\section{Introduction}

Two-dimensional quantum field theories have been a rich source of relations between different mathematical disciplines. A prominent class of examples of such theories are the two-dimensional rational conformal field theories, which admit a mathematically precise description (see [SFR for a summary of recent progress). A large subclass of these also have a classical description in terms of an action, in which a term given by a surface holonomy enters.

The appropriate geometric object for the definition of surface holonomies for oriented surfaces with empty boundary are hermitian bundle gerbes. We systematically introduce bundle gerbes by first defining a pre-stack of trivial bundle gerbes, in such a way that surface holonomy can be defined, and then closing this pre-stack under descent. This construction constitutes in fact a generalization of the geometry of line bundles, their holonomy and their applications to classical particle mechanics.

Inspired by results in a representation theoretic approach to rational conformal field theories, we then introduce in the same spirit geometric structure that allows to define surface holonomy in more general situations: Jandl gerbes for unoriented surfaces, D-branes for surfaces with boundaries, and bi-branes for surfaces with defect lines.

\section{Hermitian line bundles and holonomy}

Before discussing bundle gerbes, it is appropriate to summarize some pertinent aspects of line bundles.

One of the basic features of a (complex) line bundle $L$ over a smooth manifold $M$ is that it is locally trivializable. This means that $M$ can be covered by open sets $U_{\alpha}$ such that there exist isomorphisms $\phi_{\alpha}:\left.L\right|_{U_{\alpha}} \rightarrow \mathbf{1}_{U_{\alpha}}$, where $\mathbf{1}_{U_{\alpha}}$ denotes the trivial line bundle $\mathbb{C} \times U_{\alpha}$. A choice of such maps $\phi_{\alpha}$ defines gluing isomorphisms

$$
g_{\alpha \beta}:\left.\left.\quad \mathbf{1}_{U_{\alpha}}\right|_{U_{\alpha} \cap U_{\beta}} \rightarrow \mathbf{1}_{U_{\beta}}\right|_{U_{\alpha} \cap U_{\beta}} \quad \text { with } \quad g_{\beta \gamma} \circ g_{\alpha \beta}=g_{\alpha \gamma} \quad \text { on } U_{\alpha} \cap U_{\beta} \cap U_{\gamma} .
$$

Isomorphisms between trivial line bundles are just smooth functions. Given a set of gluing isomorphisms one can obtain as additional structure the total space as the manifold

$$
L:=\bigsqcup_{\alpha} \mathbf{1}_{U_{\alpha}} / \sim
$$

with the relation $\sim$ identifying an element $\ell$ of $\mathbf{1}_{U_{\alpha}}$ with $g_{\alpha \beta}(\ell)$ of $\mathbf{1}_{U_{\beta}}$. In short, every bundle is glued together from trivial bundles.

In the following all line bundles will be equipped with a hermitian metric, and all isomorphisms are supposed to be isometries. Such line bundles form categories, denoted $\mathcal{B} u n(M)$. The trivial bundle $\mathbf{1}_{M}$ defines a full, one-object subcategory $\mathcal{B}$ untriv $(M)$ whose endomorphism set is the monoid of $\mathrm{U}(1)$-valued functions on $M$. Denoting by $\pi_{0}(\mathcal{C})$ the set of isomorphism classes of a category $\mathcal{C}$ and by $H^{\bullet}(M, \mathrm{U}(1))$ the sheaf cohomology of $M$ with coefficients in the sheaf of $U(1)$-valued functions, we have the bijection

$$
\pi_{0}(\mathcal{B} u n(M)) \cong H^{1}(M, \underline{\mathrm{U}(1)}) \cong H^{2}(M, \mathbb{Z})
$$

under which the isomorphism class of the trivial bundle is mapped to zero. 
Another basic feature of line bundles is that they pull back along smooth maps: for $L$ a line bundle over $M$ and $f: M^{\prime} \rightarrow M$ a smooth map, the pullback $f^{*} L$ is a line bundle over $M^{\prime}$, and this pullback $f^{*}$ extends to a functor

$$
f^{*}: \quad \mathcal{B u n}(M) \rightarrow \mathcal{B} \text { un }\left(M^{\prime}\right)
$$

Furthermore, there is a unique isomorphism $g^{*}\left(f^{*} L\right) \rightarrow(f \circ g)^{*} L$ for composable maps $f$ and $g$.

As our aim is to discuss holonomies, we should in fact consider a different category, namely line bundles equipped with (metric) connections. These form again a category, denoted by $\mathcal{B}_{u n} \nabla(M)$, and this has again a full subcategory $\mathcal{B u n t r i v}^{\nabla}(M)$ of trivial line bundles with connection. But now this subcategory has more than one object: every 1-form $\omega \in \Omega^{1}(M)$ can serve as a connection on a trivial line bundle $\mathbf{1}$ over $M$; the so obtained objects are denoted by $\mathbf{1}_{\omega}$. The set $\operatorname{Hom}\left(\mathbf{1}_{\omega}, \mathbf{1}_{\omega^{\prime}}\right)$ of connection-preserving isomorphisms $\eta: \mathbf{1}_{\omega} \rightarrow \mathbf{1}_{\omega^{\prime}}$ is the set of smooth functions $g: M \rightarrow \mathrm{U}(1)$ satisfying

$$
\omega^{\prime}-\omega=-\mathrm{i} \operatorname{dlog} g
$$

Just like in (2.2), every line bundle $L$ with connection can be glued together from line bundles $\mathbf{1}_{\omega_{\alpha}}$ along connection-preserving gluing isomorphisms $\eta_{\alpha \beta}$.

The curvature of a trivial line bundle $\mathbf{1}_{\omega}$ is $\operatorname{curv}\left(\mathbf{1}_{\omega}\right):=\mathrm{d} \omega \in \Omega^{2}(M)$, and is thus invariant under connection-preserving isomorphisms. It follows that the curvature of any line bundle with connection is a globally well-defined, closed 2-form. We recall that the cohomology class of this 2-form in real cohomology coincides with the characteristic class in (2.3).

In order to introduce the holonomy of line bundles with connection, we say that the holonomy of a trivial line bundle $\mathbf{1}_{\omega}$ over $S^{1}$ is

$$
\operatorname{Hol}_{\mathbf{1}_{\omega}}:=\exp \left(2 \pi \mathrm{i} \int_{S^{1}} \omega\right) \in \mathrm{U}(1)
$$

If $\mathbf{1}_{\omega}$ and $\mathbf{1}_{\omega^{\prime}}$ are trivial line bundles over $S^{1}$, and if there exists a morphism $\eta$ in $\operatorname{Hom}\left(\mathbf{1}_{\omega}, \mathbf{1}_{\omega^{\prime}}\right)$, we have $\mathrm{Hol}_{\mathbf{1}_{\omega}}=\mathrm{Hol}_{\mathbf{1}_{\omega^{\prime}}}$ because

$$
\int_{S^{1}} \omega^{\prime}-\int_{S^{1}} \omega=\int_{S^{1}}-\mathrm{i} \operatorname{dlog} \eta \in \mathbb{Z} .
$$

More generally, if $L$ is any line bundle with connection over $M$, and $\Phi: S^{1} \rightarrow M$ is a smooth map, then the pullback bundle $\Phi^{*} L$ is trivial since $H^{2}\left(S^{1}, \mathbb{Z}\right)=0$, and hence one can choose an isomorphism $\mathcal{T}: \Phi^{*} L \stackrel{\sim}{\longrightarrow} \mathbf{1}_{\omega}$ for some $\omega \in \Omega^{1}\left(S^{1}\right)$. We then set

$$
\operatorname{Hol}_{L}(\Phi):=\operatorname{Hol}_{\mathbf{1}_{\omega}} \text {. }
$$

This is well-defined because any other trivialization $\mathcal{T}^{\prime}: \Phi^{*} L \rightarrow 1_{\omega^{\prime}}$ provides a transition isomorphism $\eta:=\mathcal{T}^{\prime} \circ \mathcal{T}^{-1}$ in $\operatorname{Hom}\left(\mathbf{1}_{\omega}, \mathbf{1}_{\omega^{\prime}}\right)$. But as we have seen above, the holonomies of isomorphic trivial line bundles coincide.

Let us also mention an elementary example of a physical application of line bundles and their holonomies: the action functional $S$ for a charged point particle. For $(M, g)$ a 
(pseudo-)Riemannian manifold and $\Phi: \mathbb{R} \supset\left[t_{1}, t_{2}\right] \rightarrow(M, g)$ the trajectory of a point particle of mass $m$ and electric charge $e$, one commonly writes the action $S[\Phi]$ as the sum of the kinetic term

$$
S_{\text {kin }}[\Phi]=\frac{m}{2} \int_{t_{1}}^{t_{2}} g\left(\frac{\mathrm{d} \Phi}{\mathrm{d} t}, \frac{\mathrm{d} \Phi}{\mathrm{d} t}\right)
$$

and a term

$$
-e \int_{t_{1}}^{t_{2}} \Phi^{*} A
$$

with $A$ the electromagnetic gauge potential. However, this formulation is inappropriate when the electromagnetic field strength $F$ is not exact, so that a gauge potential $A$ with $\mathrm{d} A=F$ exists only locally. As explained above, keeping track of such local 1-forms $A_{\alpha}$ and local 'gauge transformations', i.e. connection-preserving isomorphisms between those, leads to the notion of a line bundle $L$ with connection. For a closed trajectory, i.e. $\Phi\left(t_{1}\right)=\Phi\left(t_{2}\right)$, the action should be defined as

$$
\mathrm{e}^{\mathrm{i} S[\Phi]}=\mathrm{e}^{\mathrm{i} S_{\text {kin }}[\Phi]} \operatorname{Hol}_{L}(\Phi) .
$$

An important feature of bundles in physical applications is the 'Dirac quantization' condition on the field strength $F$ : the integral of $F$ over any closed surface $\Sigma$ in $M$ gives an integer. This follows from the coincidence of the cohomology class of $F$ with the characteristic class in (2.3) . Another aspect is a neat explanation of the Aharonov-Bohm effect. A line bundle over a nonsimply connected manifold can have vanishing curvature and yet non-trivial holonomies. In the quantum theory holonomies are observable, and thus the gauge potential $A$ contains physically relevant information even if its field strength is zero. Both aspects, the quantization condition and the Aharonov-Bohm effect, persist in the generalization of line bundles to bundle gerbes, which we discuss next.

\section{Gerbes and surface holonomy}

In this section we formalize the procedure of Section 2 that has lead us from local 1-form gauge potentials to line bundles with connection: we will explain that it is the closure of the category of trivial bundles with connection under descent. We then apply the same principle to locally defined 2-forms, whereby we arrive straightforwardly at the notion of bundle gerbes with connection. We describe the notion of surface holonomy of such gerbes and their applications to physics analogously to Section 2 .

\subsection{Descent of bundles}

As a framework for structures with a category assigned to every manifold and consistent pullback functors we consider presheaves of categories. Let Man be the category of smooth manifolds and smooth maps, and let $\mathcal{C}$ at be the 2-category of categories, with functors between categories as 1-morphisms and natural transformations between functors as 2-morphisms. Then a presheaf of categories is a lax functor

$$
\mathcal{F}: \quad \operatorname{Man}^{\text {opp }} \rightarrow \mathcal{C} \text { at }
$$


It assigns to every manifold $M$ a category $\mathcal{F}(M)$, and to every smooth map $f: M^{\prime} \rightarrow M$ a functor $\mathcal{F}(f): \mathcal{F}(M) \rightarrow \mathcal{F}\left(M^{\prime}\right)$. By the qualification 'lax' we mean that the composition of maps must only be preserved up to coherent isomorphisms.

In Section 2 we have already encountered four examples of presheaves: the presheaf $\mathcal{B} u n$ of line bundles, the presheaf $\mathcal{B} u{ }^{\nabla}$ of line bundles with connection, and their sub-presheaves of trivial bundles.

To formulate a gluing condition for presheaves of categories we need to specify coverings. Here we choose surjective submersions $\pi: Y \rightarrow M$. We remark that every cover of $M$ by open sets $U_{\alpha}$ provides a surjective submersion with $Y$ the disjoint union of the $U_{\alpha}$; thus surjective submersions generalize open coverings. This generalization proves to be important for many examples of bundle gerbes, such as the lifting of bundle gerbes and the canonical bundle gerbes of compact simple Lie groups.

With hindsight, a choice of coverings endows the category Man with a Grothendieck topology. Both surjective submersions and open covers define a Grothendieck topology, and since every surjective submersion allows for local sections, the resulting two Grothendieck topologies are equivalent. And in fact the submersion topology is the maximal one equivalent to open coverings.

Along with a covering $\pi: Y \rightarrow M$ there comes a simplicial manifold

$$
\cdots \underset{\partial_{3}}{\stackrel{\partial_{0}}{\rightleftarrows}} Y^{[3]} \underset{\partial_{2}}{\stackrel{\partial_{0}}{\rightleftarrows}} Y^{[2]} \underset{\partial_{1}}{\stackrel{\partial_{0}}{\rightleftarrows}} Y \stackrel{\pi}{\longrightarrow} M \text {. }
$$

Here $Y^{[n]}$ denotes the $n$-fold fibre product of $Y$ over $M$,

$$
Y^{[n]}:=\left\{\left(y_{0}, \ldots, y_{n-1}\right) \in Y^{n} \mid \pi\left(y_{0}\right)=\ldots=\pi\left(y_{n-1}\right)\right\},
$$

and the map $\partial_{i}: Y^{[n]} \rightarrow Y^{[n-1]}$ omits the $i$ th entry. In particular $\partial_{0}: Y^{[2]} \rightarrow Y$ is the projection to the second factor and $\partial_{1}: Y^{[2]} \rightarrow Y$ the one to the first. All fibre products $Y^{[k]}$ are smooth manifolds, and all maps $\partial_{i}$ are smooth. Now let $L$ be a line bundle over $M$. By pullback along $\pi$ we obtain:

(BO1) An object $\tilde{L}:=\pi^{*} L$ in $\mathcal{B} u n(Y)$.

(BO2) A morphism

$$
\phi: \quad \partial_{0}^{*} \tilde{L} \cong \partial_{0}^{*} \pi^{*} L \stackrel{\sim}{\longrightarrow} \partial_{1}^{*} \pi^{*} L \cong \partial_{1}^{*} \tilde{L}
$$

in $\operatorname{Bun}\left(Y^{[2]}\right)$ induced from the identity $\pi \circ \partial_{0}=\pi \circ \partial_{1}$.

(BO3) A commutative diagram

$$
\partial_{1}^{*} \partial_{0}^{*} \tilde{L}=\partial_{0}^{*} \partial_{0}^{*} \tilde{L} \stackrel{\partial_{0}^{*} \phi}{\longrightarrow} \partial_{0}^{*} \partial_{1}^{*} \tilde{L}=\partial_{2}^{*} \partial_{0}^{*} \tilde{L} \stackrel{\partial_{2}^{*} \phi}{\longrightarrow} \partial_{2}^{*} \partial_{1}^{*} \tilde{L}=\partial_{1}^{*} \partial_{1}^{*} \tilde{L}
$$

of morphisms in $\mathcal{B} u n\left(Y^{[3]}\right)$; or in short, an equality $\partial_{2}^{*} \phi \circ \partial_{0}^{*} \phi=\partial_{1}^{*} \phi$.

We call a pair $(\tilde{L}, \phi)$ as in (BO1) and (BO2) which satisfies (BO3) a descent object in the presheaf $\mathcal{B} u n$. Analogously we obtain for a morphism $f: L \rightarrow L^{\prime}$ of line bundles over $M$ 
(BM1) A morphism $\tilde{f}:=\pi^{*} f: \tilde{L} \rightarrow \tilde{L}^{\prime}$ in $\mathcal{B} u n(Y)$.

(BM2) A commutative diagram

$$
\phi^{\prime} \circ \partial_{0}^{*} \tilde{f}=\partial_{1}^{*} \tilde{f} \circ \phi
$$

of morphisms in $\mathcal{B} u n\left(Y^{[2]}\right)$.

Such a morphism $\tilde{f}$ as in (BM1) obeying (BM2) is called a descent morphism in the presheaf Bun.

Descent objects and descent morphisms for a given covering $\pi$ form a category $\operatorname{Desc}(\pi: Y \rightarrow M)$ of descent data. What we described above is a functor

$$
\iota_{\pi}: \operatorname{Bun}(M) \rightarrow \operatorname{Desc}(\pi: Y \rightarrow M)
$$

The question arises whether every 'local' descent object corresponds to a 'global' object on $M$, i.e. whether the functor $\iota_{\pi}$ is an equivalence of categories.

The construction generalizes straightforwardly to any presheaf of categories $\mathcal{F}$, and if the functor $\iota_{\pi}$ is an equivalence for all coverings $\pi: Y \rightarrow M$, the presheaf $\mathcal{F}$ is called a sheaf of categories (or stack). Extending the gluing process from (2.2) to non-trivial bundles shows that the presheaves $\mathcal{B} u n$ and $\mathcal{B} u{ }^{\nabla}$ are sheaves. In contrast, the presheaves $\mathcal{B}$ untriv and $\mathcal{B u n t r i v}^{\nabla}$ of trivial bundles are not sheaves, since gluing of trivial bundles does in general not result in a trivial bundle. In fact the gluing process (2.2) shows that every bundle can be obtained by gluing trivial ones. In short, the sheaf $\mathcal{B}^{\nabla}{ }^{\nabla}$ of line bundles with connection is obtained by closing the presheaf $\mathcal{B u n t r i v}^{\nabla}$ under descent.

\subsection{Bundle gerbes}

Our construction of line bundles started from trivial line bundles with connection which are just 1-forms on $M$, and the fact that 1-forms can be integrated along curves has lead us to the notion of holonomy. To arrive at a notion of surface holonomy, we now consider a category of 2-forms, or rather a 2-category:

- An object is a 2-form $\omega \in \Omega^{2}(M)$, called a trivial bundle gerbe with connection and denoted by $\mathcal{I}_{\omega}$.

- A 1-morphism $\eta: \omega \rightarrow \omega^{\prime}$ is a 1-form $\eta \in \Omega^{1}(M)$ such that $\mathrm{d} \eta=\omega^{\prime}-\omega$.

- A 2-morphism $\phi: \eta \Longrightarrow \eta^{\prime}$ is a smooth function $\phi: M \rightarrow \mathrm{U}(1)$ such that $-\mathrm{i} \operatorname{d} \log (\phi)=\eta^{\prime}-\eta$.

There is also a natural pullback operation along maps, induced by pullback on differential forms. The given data can be rewritten as a presheaf of 2-categories, as there is a 2-category attached to each manifold. This presheaf should now be closed under descent to obtain a sheaf of 2-categories. As a first step we complete the morphism categories under descent. Since these are categories of trivial line bundles with connections, we set

$$
\operatorname{Hom}\left(\mathcal{I}_{\omega}, \mathcal{I}_{\omega^{\prime}}\right):=\mathcal{B u n}_{\omega^{\prime}-\omega}^{\nabla}(M)
$$

the category of hermitian line bundles with connection of fixed curvature $\omega^{\prime}-\omega$. The horizontal composition is given by the tensor product in the category of bundles. Finally, completing the 2-category under descent, we get the definition of a bundle gerbe: 
Definition 1. A bundle gerbe $\mathcal{G}$ (with connection) over $M$ consists of the following data: a covering $\pi: Y \rightarrow M$, and for the associated simplicial manifold

$$
Y^{[4]} \underset{\rightrightarrows}{\Longrightarrow} Y^{[3]} Y^{[2]} \underset{\partial_{1}}{\stackrel{\partial_{0}}{\rightleftarrows}} Y \stackrel{\pi}{\longrightarrow} M
$$

(GO1) an object $\mathcal{I}_{\omega}$ of $\mathcal{G}_{\operatorname{rbtriv}}^{\nabla}(Y)$ : a 2-form $\omega \in \Omega^{2}(Y)$;

(GO2) a 1-morphism

$$
L: \partial_{0}^{*} \mathcal{I}_{\omega} \rightarrow \partial_{1}^{*} \mathcal{I}_{\omega}
$$

in $\mathcal{G r b t r i v}^{\nabla}\left(Y^{[2]}\right)$ : a line bundle $L$ with connection over $Y^{[2]}$;

(GO3) a 2-isomorphism

$$
\mu: \partial_{2}^{*} L \otimes \partial_{0}^{*} L \Longrightarrow \partial_{1}^{*} L
$$

in $\mathcal{G r b t r i v}^{\nabla}\left(Y^{[3]}\right)$ : a connection-preserving morphism of line bundles over $Y^{[3]}$;

(GO4) an equality

$$
\partial_{2}^{*} \mu \circ\left(\mathrm{id} \otimes \partial_{0}^{*} \mu\right)=\partial_{1}^{*} \mu \circ\left(\partial_{3}^{*} \mu \otimes \mathrm{id}\right)
$$

of 2-morphisms in $\mathcal{G r b t r i v}^{\nabla}\left(Y^{[4]}\right)$.

For later applications it will be necessary to close the morphism categories under a second operation, namely direct sums. Closing the category of line bundles with connection under direct sums leads to the category of complex vector bundles with connection, i.e. we set

$$
\operatorname{Hom}\left(\mathcal{I}_{\omega}, \mathcal{I}_{\omega^{\prime}}\right):=\operatorname{Vect} \mathcal{B u n}_{\omega^{\prime}-\omega}^{\nabla}(M),
$$

where the curvature of these vector bundles is constrained to satisfy

$$
\frac{1}{n} \operatorname{tr}(\operatorname{curv}(L))=\omega^{\prime}-\omega,
$$

with $n$ the rank of the vector bundle. Notice that this does not affect the definition of a bundle gerbe, since the existence of the 2-isomorphism $\mu$ restricts the rank of $L$ to be one.

As a next step, we need to introduce 1-morphisms and 2-morphisms between bundle gerbes. 1-morphisms have to compare two bundle gerbes $\mathcal{G}$ and $\mathcal{G}^{\prime}$. We assume first that both bundle gerbes have the same covering $Y \rightarrow M$.

Definition 2. i) A 1-morphism between bundle gerbes $\mathcal{G}=(Y, \omega, L, \mu)$ and $\mathcal{G}^{\prime}=\left(Y, \omega^{\prime}, L^{\prime}, \mu^{\prime}\right)$ over $M$ with the same surjective submersion $Y \rightarrow M$ consists of the following data on the associated simplicial manifold

$$
Y^{[4]} \underset{\rightrightarrows}{\rightrightarrows} Y^{[3]} \Longrightarrow Y^{[2]} \underset{\partial_{1}}{\stackrel{\partial_{0}}{\rightleftarrows}} Y \stackrel{\pi}{\longrightarrow} M
$$

(G1M1) a 1-morphism $A: \mathcal{I}_{\omega} \rightarrow \mathcal{I}_{\omega^{\prime}}$ in $\mathcal{G}_{\operatorname{rbtriv}}{ }^{\nabla}(Y)$ : a rank- $n$ hermitian vector bundle $A$ with connection of curvature $\frac{1}{n} \operatorname{tr}(\operatorname{curv}(L))=\omega^{\prime}-\omega$;

(G1M2) a 2-isomorphism $\alpha: L^{\prime} \otimes \partial_{0}^{*} A \Longrightarrow \partial_{1}^{*} A \otimes L$ in $\mathcal{G r b t r i v}^{\nabla}\left(Y^{[2]}\right)$ : a connection-preserving morphism of hermitian vector bundles; 
(G1M3) a commutative diagram

$$
\left(\mathrm{id} \otimes \mu^{\prime}\right) \circ\left(\partial_{2}^{*} \alpha \otimes \mathrm{id}\right) \circ\left(\mathrm{id} \otimes \partial_{0}^{*} \alpha\right)=\partial_{1}^{*} \alpha \circ(\mu \otimes i d)
$$

of 2-morphisms in $\mathcal{G r b t r i v}^{\nabla}\left(Y^{[3]}\right)$.

ii) A 2-morphism between two such 1-morphisms $(A, \alpha)$ and $\left(A^{\prime}, \alpha^{\prime}\right)$ consists of

(G2M1) a 2-morphism $\beta: A \Longrightarrow A^{\prime}$ in $\mathcal{G}_{\operatorname{rbtriv}} \nabla(Y)$ : a connection-preserving morphism of vector bundles;

(G2M2) a commutative diagram

$$
\alpha^{\prime} \circ\left(\mathrm{id} \otimes \partial_{0}^{*} \beta\right)=\left(\partial_{1}^{*} \beta \otimes \mathrm{id}\right) \circ \alpha
$$

of 2-morphisms in $\mathcal{G r b t r i v}^{\nabla}\left(Y^{[2]}\right)$.

Since 1-morphisms are composed by taking tensor products of vector bundles, a 1-morphism is invertible if and only if its vector bundle is of rank one.

In order to define 1-morphisms and 2-morphisms between bundle gerbes with possibly different coverings $\pi: Y \rightarrow M$ and $\pi^{\prime}: Y^{\prime} \rightarrow M$, we pull all the data back to a common refinement of these coverings and compare them there. We call a covering $\zeta: Z \rightarrow M$ a common refinement of $\pi$ and $\pi^{\prime}$ iff there exist maps $s: Z \rightarrow Y$ and $s^{\prime}: Z \rightarrow Y^{\prime}$ such that

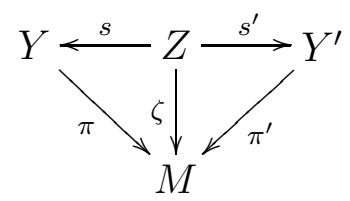

commutes. An example of such a common refinement is the fibre product $Z:=Y \times_{M} Y^{\prime} \rightarrow M$, with the maps $Z \rightarrow Y$ and $Z \rightarrow Y^{\prime}$ given by the projections. The important point about a common refinement $Z \rightarrow M$ is that the maps $s$ and $s^{\prime}$ induce simplicial maps

$$
Y^{\bullet} \longleftarrow Z^{\bullet} \longrightarrow Y^{\bullet}
$$

For bundle gerbes $\mathcal{G}=(Y, \omega, L, \mu)$ and $\mathcal{G}^{\prime}=\left(Y^{\prime}, \omega^{\prime}, L^{\prime}, \mu^{\prime}\right)$ we obtain new bundle gerbes with surjective submersion $Z$ by pulling back all the data along the simplicial maps $s$ and $s^{\prime}$. Explicitly, $\mathcal{G}_{Z}:=\left(Z, s_{0}^{*} \omega, s_{1}^{*} L, s_{2}^{*} \mu\right)$ and $\mathcal{G}_{Z}^{\prime}=\left(Z, s_{0}^{* *} \omega^{\prime}, s_{1}^{\prime *} L^{\prime}, s_{2}^{* *} \mu^{\prime}\right)$. Also morphisms can be refined by pulling them back.

Definition 3. i) A 1-morphism between bundle gerbes $\mathcal{G}=(Y, \omega, L, \mu)$ and $\mathcal{G}^{\prime}=\left(Y^{\prime}, \omega^{\prime}, L^{\prime}, \mu^{\prime}\right)$ consists of a common refinement $Z \rightarrow M$ of the coverings $Y \rightarrow M$ and $Y^{\prime} \rightarrow M$ and a morphism $(A, \alpha)$ of the two refined gerbes $\mathcal{G}_{Z}$ and $\mathcal{G}_{Z}^{\prime}$.

ii) A 2-morphism between 1-morphisms $\mathfrak{m}=(Z, A, \alpha)$ and $\mathfrak{m}^{\prime}=\left(Z^{\prime}, A^{\prime}, \alpha^{\prime}\right)$ consists of a common refinement $W \rightarrow M$ of the coverings $Z \rightarrow M$ and $Z^{\prime} \rightarrow M$ (respecting the projections to $Y$ and $Y^{\prime}$, respectively) and a 2-morphism $\beta$ of the refined morphisms $\mathfrak{m}_{W}$ and $\mathfrak{m}_{W}^{\prime}$. In addition two such 2-morphisms $(W, \beta)$ and $\left(W^{\prime}, \beta^{\prime}\right)$ must be identified iff there exists a further common refinement $V \rightarrow M$ of $W \rightarrow M$ and $W^{\prime} \rightarrow M$, compatible with the other projections, such that the refined 2-morphisms agree on $V$. 
For a gerbe $\mathcal{G}=(Y, \omega, L, \mu)$ and a refinement $Z \rightarrow M$ of $Y$ the refined gerbe $\mathcal{G}_{Z}$ is isomorphic to $\mathcal{G}$. This implies that every gerbe is isomorphic to a gerbe defined over an open covering $Z:=\bigsqcup_{i \in I} U_{i}$. Furthermore we can choose the covering in such a way that the line bundle over double intersections is trivial as well. When doing so we obtain the familiar description of gerbes in terms of local data, reproducing formulas by [Al, Ga1. Extending this description to morphisms it is straightforward to show that gerbes are classified by the so-called Deligne cohomology $H^{k}(M, \mathcal{D}(2))$ in degree two:

$$
\pi_{0}\left(\mathcal{G r b}^{\nabla}(M)\right) \cong H^{2}(M, \mathcal{D}(2))
$$

Analogously we get the classification of gerbes without connection as

$$
\pi_{0}(\mathcal{G} r b(M)) \cong H^{2}(M, \underline{\mathrm{U}(1)}) \cong H^{3}(M, \mathbb{Z})
$$

\subsection{Surface holonomy}

The holonomy of a trivial bundle gerbe $\mathcal{I}_{\omega}$ over a closed oriented surface $\Sigma$ is by definition

$$
\operatorname{Hol}_{\mathcal{I}_{\omega}}:=\exp \left(2 \pi \mathrm{i} \int_{\Sigma} \omega\right) \in \mathrm{U}(1)
$$

If $\mathcal{I}_{\omega}$ and $\mathcal{I}_{\omega^{\prime}}$ are two trivial bundle gerbes over $\Sigma$ such that there exists a 1-isomorphism $\mathcal{I}_{\omega} \rightarrow I_{\omega^{\prime}}$, i.e. a vector bundle $L$ of rank one, we have an equality $\operatorname{Hol}_{\mathcal{I}_{\omega}}=\operatorname{Hol}_{\mathcal{I}_{\omega^{\prime}}}$ because

$$
\int_{\Sigma} \omega^{\prime}-\int_{\Sigma} \omega=\int_{\Sigma} \operatorname{curv}(L) \in \mathbb{Z}
$$

More generally, consider a bundle gerbe $\mathcal{G}$ with connection over a smooth manifold $M$, and a smooth map

$$
\Phi: \quad \Sigma \rightarrow M
$$

defined on a closed oriented surface $\Sigma$. Since $H^{3}(\Sigma, \mathbb{Z})=0$, the pullback $\Phi^{*} \mathcal{G}$ is isomorphic to a trivial bundle gerbe. Hence one can choose a trivialization, i.e. a 1-isomorphism

$$
\mathcal{T}: \quad \Phi^{*} \mathcal{G} \stackrel{\sim}{\longrightarrow} \mathcal{I}_{\omega}
$$

and define the holonomy of $\mathcal{G}$ around $\Phi$ by

$$
\operatorname{Hol}_{\mathcal{G}}(\Phi):=\operatorname{Hol}_{\mathcal{I}_{\omega}}
$$

In the same way as for the holonomy of a line bundle with connection, this definition is independent of the choice of the 1-isomorphism $\mathcal{T}$. Namely, if $\mathcal{T}^{\prime}: \Phi^{*} \mathcal{G} \stackrel{\sim}{\longrightarrow} \mathcal{I}_{\omega^{\prime}}$ is another trivialization, we have a transition isomorphism

$$
L:=\mathcal{T}^{\prime} \circ \mathcal{T}^{-1}: \quad \mathcal{I}_{\omega} \stackrel{\sim}{\longrightarrow} \mathcal{I}_{\omega^{\prime}},
$$

which shows the independence. 


\subsection{Wess-Zumino terms}

As we have seen in Section 2, the holonomy of a line bundle with connection supplies a term in the action functional of a classical charged particle, describing the coupling to a gauge field whose field strength is the curvature of the line bundle. Analogously, the surface holonomy of a bundle gerbe with connection defines a term in the action of a classical charged string. Such a string is described in terms of a smooth map $\Phi: \Sigma \rightarrow M$. The exponentiated action functional of the string is (compare (2.11) $)$

$$
\mathrm{e}^{\mathrm{i} S[\Phi]}=\mathrm{e}^{\mathrm{i} S_{\text {kin }}[\Phi]} \operatorname{Hol}_{\mathcal{G}}(\Phi)
$$

where $S_{\text {kin }}[\Phi]$ is a kinetic term which involves a conformal structure on $\Sigma$. Physical models whose fields are maps defined on surfaces are called (non-linear) sigma models, and the holonomy term is called a Wess-Zumino term. Such terms are needed in certain models in order to obtain quantum field theories that are conformally invariant.

A particular class of sigma models with Wess-Zumino term is given by WZW (Wess-ZuminoWitten) models. For these the target space $M$ is a connected compact simple Lie group $G$, and the curvature of the bundle gerbe $\mathcal{G}$ is an integral multiple of the canonical 3-form

$$
H=\langle\theta \wedge[\theta \wedge \theta]\rangle \in \Omega^{3}(G)
$$

( $\theta$ is the left-invariant Maurer-Cartan form on $G$, and $\langle\cdot, \cdot\rangle$ the Killing form of the Lie algebra $\mathfrak{g}$ of $G$ ). WZW models have been a distinguished arena for the interplay between Lie theory and the theory of bundle gerbes [Ga1, GR]. This has lead to new insights both in the physical applications and in the underlying mathematical structures. Some of these will be discussed in the following sections.

Defining Wess-Zumino terms as the holonomy of a bundle gerbe with connection allows one in particular to explain the following two facts.

- The Aharonov-Bohm effect: This occurs when the bundle gerbe has a flat connection, i.e. its curvature $H \in \Omega^{3}(M)$ vanishes. This does not mean, though, that the bundle gerbe is trivial, since its class in $H^{3}(M, \mathbb{Z})$ may be pure torsion. In particular, it can still have non-constant holonomy, and thus a non-trivial Wess-Zumino term.

An example for the Aharonov-Bohm effect is the sigma model on the 2-torus $T=S^{1} \times S^{1}$. By dimensional reasons, the 3 -form $H$ vanishes. Nonetheless, since $H^{2}(T, \mathrm{U}(1))=\mathrm{U}(1)$, there exists a whole family of Wess-Zumino terms parameterized by an angle, of which only the one with angle zero is trivial.

- Discrete torsion: The set of isomorphism classes of bundle gerbes with connection that have the same curvature $H$ is parameterized by $H^{2}(M, \mathrm{U}(1))$ via the map

$$
H^{2}(M, \mathrm{U}(1)) \rightarrow \operatorname{Tors}\left(H^{3}(M, \mathbb{Z})\right) .
$$

If this group is non-trivial, there exist different Wess-Zumino terms for one and the same field strength $H$; their difference is called 'discrete torsion'.

An example for discrete torsion is the level- $k$ WZW model on the Lie group $\operatorname{PSO}(4 n)$. Since $H^{2}(\mathrm{PSO}(4 n), \mathrm{U}(1))=\mathbb{Z}_{2}$, there exist two non-isomorphic bundle gerbes with connection having equal curvature. 


\section{The representation theoretic formulation of RCFT}

\subsection{Sigma models}

Closely related to surface holonomies are novel geometric structures that have been introduced for unoriented surfaces, for surfaces with boundary, and for surfaces with defect lines. These structures constitute the second theme of this contribution, extending the construction of gerbes and surface holonomy via descent; they will be discussed in Sections 5, 6 and 7 .

These geometric developments were in fact strongly inspired by algebraic and representation theoretic results in two-dimensional quantum field theories. To appreciate this connection we briefly review in this section the relation between spaces of maps $\Phi: \Sigma \rightarrow M$, as they appear in the treatment of holonomies, and quantum field theories.

As already indicated in Section [3.4, a classical field theory, the (non-linear) sigma model, on a two-dimensional surface $\Sigma$, called the world sheet, can be associated to the space of smooth maps $\Phi$ from $\Sigma$ to some smooth manifold $M$, called the target space. Appropriate structure on the target space determines a Lagrangian for the field theory on $\Sigma$. Geometric structure on $M$, e.g. a (pseudo-) Riemannian metric $G$, becomes, from this point of view, for any given map $\Phi$ a background function $G(\Phi(x))$ for the field theory on $\Sigma$.

Three main issues will then lead us to a richer structure related to surface holonomies:

- In string theory (where the world sheet $\Sigma$ arises as the surface swept out by a string moving in $M$ ) and in other applications as well, one also encounters sigma models on world sheets $\Sigma$ that have non-empty boundary. We will explain how the geometric data relevant for encoding boundary conditions - so called D-branes - can be derived from geometric principles.

- String theories of type I, which form an integral part of string dualities, involve unoriented world sheets. In string theory it is therefore a fundamental problem to exhibit geometric structure on the target space that provides a notion of holonomy for unoriented surfaces.

- An equally natural structure present in quantum field theory are topological defect lines, along which correlation functions of bulk fields can have a branch-cut. In specific models these can be understood, just like boundary conditions, as continuum versions of corresponding structures in lattice models of statistical mechanics. (For instance, in the lattice version of the Ising model a topological defect is produced by changing the coupling along all bonds that cross a specified line from ferromagnetic to antiferromagnetic.)

Sigma models have indeed been a significant source of examples for quantum field theories, at least on a heuristic level. Conversely, having a sigma model interpretation for a given quantum field theory allows for a geometric interpretation of quantum field theoretic quantities. Sigma models have indeed been, at least on a heuristic level, a significant source of examples for quantum field theories. Conversely, quantum field theoretic structures in sigma models have lead to structural insights and quantitative predictions in geometry.

A distinguished subclass of theories in which this relationship between quantum field theory and geometry can be studied are two-dimensional conformal field theories, or CFTs, for short, and among these in particular the rational conformal field theories for which there exists a rigorous representation theoretic approach. The structures appearing in that approach in the three situations mentioned above suggest new geometric notions for conformal sigma models. Below we will investigate these notions with the help of standard geometric principles. Before 
doing so we formulate, in representation theoretic terms, the relevant aspects of the quantum field theories in question.

\subsection{Rational conformal field theory}

The conformal symmetry, together with further, so-called chiral, symmetries of a CFT can be encoded in the structure of a conformal vertex algebra $\mathcal{V}$. For any conformal vertex algebra one can construct (see e.g. [FrB]) a chiral CFT; in mathematical terms, a chiral CFT is a system of conformal blocks, i.e. sheaves over the moduli spaces of curves with marked points. These sheaves of conformal blocks are endowed with a projectively flat connection, the Knizhnik-Zamolodchikov connection, which in turn furnishes representations of the fundamental groups of the moduli spaces, i.e. of the mapping class groups.

Despite the physical origin of its name, a chiral conformal field theory is mathematically rigorous. On the other hand, from the two-dimensional point of view it is, despite its name, not a conventional quantum field theory, as one deals with (sections of) bundles instead of local correlation functions. In particular, it must not be confused with a full local conformal field theory, which is the relevant structure to enter our discussion of holonomies.

Chiral conformal field theories are particularly tractable when the vertex algebra $\mathcal{V}$ is rational in the sense of [Hu, thm 2.1]. Then the representation category $\mathcal{C}$ of $\mathcal{V}$ is a modular tensor category, and the associated chiral CFT is a rational chiral CFT, or chiral RCFT. In this situation, we can use the tools of three-dimensional topological quantum field theory (TFT). A TFT is, in short, a monoidal functor $\operatorname{tft}_{\mathcal{C}}[\mathrm{Tu}$, chap. IV.7] that associates a finite-dimensional vector space $\operatorname{tft}_{\mathcal{C}}(E)$ to any (extended) surface $E$, and a linear map from $\operatorname{tft}_{\mathcal{C}}(E)$ to $\operatorname{tft}_{\mathcal{C}}\left(E^{\prime}\right)$ to any (extended) cobordism $\mathcal{M}: \mathrm{E} \rightarrow \mathrm{E}^{\prime}$.

More precisely, a three-dimensional TFT is a projective monoidal functor from a category $\mathcal{C} b_{\mathcal{C}}$ of decorated cobordisms to the category of finite-dimensional complex vector spaces. The modular tensor category $\mathcal{C}$ provides the decoration data for $\mathcal{C}_{0} b_{\mathcal{C}}$. Specifically, the objects $\mathrm{E}$ of $\mathcal{C}_{0} b_{\mathcal{C}}$ are extended surfaces, i.e.1 1 compact closed oriented two-manifolds with a finite set of embedded arcs, and each of these arcs is marked by an object of $\mathcal{C}$. A morphism $\mathrm{E} \rightarrow \mathrm{E}^{\prime}$ is an extended cobordism, i.e. a compact oriented three-manifold $\mathcal{M}$ with $\partial \mathcal{M}=(-\mathrm{E}) \sqcup \mathrm{E}^{\prime}$, together with an oriented ribbon graph $\Gamma_{\mathcal{M}}$ in $\mathcal{M}$ such that at each marked arc of $(-\mathrm{E}) \sqcup \mathrm{E}^{\prime}$ a ribbon of $\Gamma_{\mathcal{M}}$ is ending. Each ribbon of $\Gamma_{\mathcal{M}}$ is labeled by an object of $\mathcal{C}$, while each coupon of $\Gamma_{\mathcal{M}}$ is labeled by an element of the morphism space of $\mathcal{C}$ that corresponds to the objects of the ribbons which enter and leave the coupon. Composition in $\mathcal{C o b}_{\mathcal{C}}$ is defined by gluing, the identity morphism $\mathrm{id}_{\mathrm{E}}$ is the cylinder over $\mathrm{E}$, and the tensor product is given by disjoint union of objects and cobordisms.

A topological field theory furnishes, for any extended surface, a representation of the mapping class group. Our approach relies on the fundamental conjecture (which is largely established for a broad class of models) that, for $\mathcal{C}$ the representation category of a rational vertex algebra $\mathcal{V}$, the mapping class group representation given by $\operatorname{tft}_{\mathcal{C}}$ is equivalent to the one provided by the Knizhnik-Zamolodchikov connection on the conformal blocks for the vertex algebra V.

\footnotetext{
${ }^{1}$ Here various details are suppressed. Detailed information, e.g. the precise definition of a ribbon graph or the reason why $\operatorname{tft}_{\mathcal{C}}$ is only projective, can be found in many places, such as [Tu, BK, KRT] or [FFFS, sect. 2.5-2.7].
} 


\subsection{The TFT construction of full RCFT}

Let us now turn to the discussion of full local conformal field theories, which are the structures to be compared to holonomies. A full CFT is, by definition, a consistent system of local correlation functions that satisfy all sewing constraints (see e.g. [FjFRS2, def. 3.14]). According to the principle of holomorphic factorization, every full RCFT can be understood with the help of a corresponding chiral CFT. The relevant chiral CFT is, however, not defined on world sheets $\Sigma$ (which may be unoriented or have a non-empty boundary), but rather on their complex doubles $\widehat{\Sigma}$, which can be given the structure of extended surfaces; this affords a geometric separation of left- and right-movers. The double $\widehat{\Sigma}$ of $\Sigma$ is, by definition, the orientation bundle over $\Sigma$ modulo identification of the two points in the fibre over each boundary point of $\Sigma$. The world sheet $\Sigma$ can be obtained from $\widehat{\Sigma}$ as the quotient by an orientation-reversing involution $\tau$. To give some examples, when $\Sigma$ is closed and orientable, then $\widehat{\Sigma}$ is just the disconnected sum $\widehat{\Sigma}=\Sigma \sqcup-\Sigma$ of two copies of $\Sigma$ with opposite orientation, and the involution $\tau$ just exchanges these two copies; the double of both the disk and the real projective plane is the two-sphere (with $\tau$ being given, in standard complex coordinates, by $z \mapsto \bar{z}^{-1}$ and by $z \longmapsto-\bar{z}^{-1}$, respectively); and the double of both the annulus and the Möbius strip is a two-torus. Further, when $\Sigma$ comes with field insertions, that is, embedded arcs labeled by objects of either $\mathcal{C}$ (for arcs on $\partial \Sigma$ ) or pairs of objects of $\mathcal{C}$ (for arcs in the interior of $\Sigma$ ), then corresponding arcs labeled by objects of $\mathcal{C}$ are present on $\widehat{\Sigma}$.

Given this connection between the surfaces relevant to chiral and full CFT, the relationship between the chiral and the full CFT can be stated as follows: A correlation function $C(\Sigma)$ of the full CFT on $\Sigma$ is a specific element in the appropriate space of conformal blocks of the chiral CFT on the double $\widehat{\Sigma}$. A construction of such elements has been accomplished in [FRS1, FRS2, FRS3, FjFRS1]. The first observation is that they can be computed with the help of the corresponding TFT, namely as

$$
C(\Sigma)=\operatorname{tft}_{\mathcal{C}}\left(\mathcal{M}_{\Sigma}\right) 1 \in \operatorname{tft}_{\mathcal{C}}(\widehat{\Sigma})
$$

Here $\mathcal{M}_{\Sigma} \equiv \emptyset \stackrel{\mathcal{M}_{\Sigma}}{\longrightarrow} \widehat{\Sigma}$, the connecting manifold for the world sheet $\Sigma$, is an extended cobordism that is constructed from the data of $\Sigma$. Besides the category $\mathcal{C}$, the specification of the vector $C(\Sigma)$ needs a second ingredient: a (Morita class of a) symmetric special Frobenius algebra $A$ in $\mathcal{C}$.

Let us give some details 2 of the construction of $C(\Sigma)$.

- As a three-manifold, $\mathcal{M}_{\Sigma}$ is the interval bundle over $\Sigma$ modulo a $\mathbb{Z}_{2}$-identification of the intervals over $\partial \Sigma$. Explicitly,

$$
\mathcal{M}_{\Sigma}=(\widehat{\Sigma} \times[-1,1]) / \sim \quad \text { with } \quad\left(\left[x, \mathrm{or}_{2}\right], t\right) \sim\left(\left[x,-\mathrm{or}_{2}\right],-t\right) .
$$

It follows in particular that $\partial \mathcal{M}_{\Sigma}=\widehat{\Sigma}$ and that $\Sigma$ is naturally embedded in $\mathcal{M}_{\Sigma}$ as $\imath: \Sigma \stackrel{\simeq}{\longrightarrow} \Sigma \times\{t=0\} \hookrightarrow \mathcal{M}_{\Sigma}$. Indeed, $\imath(\Sigma)$ is a deformation retract of $\mathcal{M}_{\Sigma}$, so that the topology of $\mathcal{M}_{\Sigma}$ is completely determined by the one of $\Sigma$.

\footnotetext{
${ }^{2}$ For another brief summary, with different emphasis, see Section 7 of [FRS4]. An in-depth exposition, including for instance the relevance of various orientations, can e.g. be found in Appendix B of [FjFRS1].
} 
- A crucial ingredient of the construction of the ribbon graph $\Gamma_{\mathcal{M}_{\Sigma}}$ in $\mathcal{M}_{\Sigma}$ is a (dual) oriented triangulation $\Gamma$ of the submanifold $\imath(\Sigma)$ of $\mathcal{M}_{\Sigma}$. This triangulation is labeled by objects and morphisms of $\mathcal{C}$. It is here that the Frobenius algebra $A$ enters: Each edge of $\Gamma \backslash \imath(\partial \Sigma)$ is covered with a ribbon labeled by the object $A$ of $\mathcal{C}$, while each (three-valent) vertex is covered with a coupon labeled by the multiplication morphism $m \in \operatorname{Hom}_{\mathcal{C}}(A \otimes A, A)$. In addition, whenever these assignments in themselves would be in conflict with the orientations of the edges, a coupon with morphism in either $\operatorname{Hom}_{\mathcal{C}}(A \otimes A, \mathbf{1})$ or $\operatorname{Hom}_{\mathcal{C}}(\mathbf{1}, A \otimes A)$ is inserted. Such morphisms are part of the data for a Frobenius structure on $A$. Assuming, for now, that the world sheet $\Sigma$ is oriented, independence of $C(\Sigma)$ from the choice of triangulation $\Gamma$ amounts precisely to the statement that the object $A$ carries the structure of a symmetric special Frobenius algebra.

- If $\Sigma$ has non-empty boundary, the prescription for $\Gamma$ is amended as follows. Each edge $e$ of $\Gamma \cap \imath(\partial \Sigma)$ is covered with a ribbon labeled by a (left, say) $A$-module $N=N(e)$, while each vertex lying on $\imath(\partial \Sigma)$ is covered with a coupon that has incoming $N$ - and $A$-ribbons as well as an outgoing $N$-ribbon and that is labeled by the representation morphism $\rho_{N} \in \operatorname{Hom}_{\mathcal{C}}(A \otimes N, N)$. The physical interpretation of the $A$-module $N$ is as the boundary condition that is associated to a component of $\partial \Sigma$. That the object $N$ of $\mathcal{C}$ labeling a boundary condition carries the structure of an $A$-module and that the morphism $\rho_{N}$ is the corresponding representation morphism is precisely what is required (in addition to $A$ being a symmetric special Frobenius algebra) in order to get independence of $C(\Sigma)$ from the choice of triangulation $\Gamma$.

- If $\Sigma$ is unoriented, then as an additional feature one must ensure independence of $C(\Sigma)$ from the choice of local orientations of $\Sigma$. As shown in [FRS2], this requires an additional structure on the algebra $A$, namely the existence of a morphism $\sigma \in \operatorname{Hom}_{\mathcal{C}}(A, A)$ that is an algebra isomorphism from the opposite algebra $A^{\text {opp }}$ to $A$ and squares to the twist of $A$, i.e. satisfies

$$
\sigma \circ \eta=\eta, \quad \sigma \circ m=m \circ c_{A, A} \circ(\sigma \otimes \sigma), \quad \sigma \circ \sigma=\theta_{A},
$$

where $\eta \in \operatorname{Hom}_{\mathcal{C}}(\mathbf{1}, A), \theta_{A} \in \operatorname{Hom}_{\mathcal{C}}(A, A)$ and $c_{A, A} \in \operatorname{Hom}_{\mathcal{C}}(A \otimes A, A \otimes A)$ denote the unit morphism, the twist, and the self-braiding of $A$, respectively. This way $A$ becomes a braided version of an algebra with involution. A symmetric special Frobenius algebra endowed with a morphism $\sigma$ satisfying (4.3) is called a Jandl algebra.

- In the presence of topological defect lines on $\Sigma$ a further amendment of the prescription is in order. The defect lines partition $\Sigma$ into disjoint regions, and to the regions to the left and to the right of a defect line one may associate different (symmetric special Frobenius) algebras $A_{l}$ and $A_{r}$, such that the part of the triangulation $\Gamma$ in one region is labeled by the algebra $A_{l}$, while the part of $\Gamma$ in the other region is labeled by $A_{r}$. The defect lines are to be regarded as forming a subset $\Gamma^{D}$ of $\Gamma$ themselves; each edge of $\Gamma^{D}$ is covered with a ribbon labeled by some object $B$ of $\mathcal{C}$, while each vertex of $\Gamma$ lying on $\Gamma^{D}$ is covered with a coupon labeled by a morphism $\rho \in \operatorname{Hom}_{\mathcal{C}}\left(A_{l} \otimes B, B\right)$, respectively $q \in \operatorname{Hom}_{\mathcal{C}}\left(B \otimes A_{r}, B\right)$. Consistency requires that these morphisms endow the object $B$ of $\mathcal{C}$ that labels a defect line with the structure of an $A_{l}$ - $A_{r}$-bimodule. (Below we will concentrate on the case $A_{l}=A_{r}=: A$, so that we deal with $A$-bimodules.) 
- There are also rules for the morphisms of $\mathcal{C}$ that label bulk, boundary and defect fields, respectively.

The prescription summarized above allows one to construct the correlator (4.1) for any arbitrary world sheet $\Sigma$. The so obtained correlators can be proven [FjFRS1 to satisfy all consistency conditions that the correlators of a CFT must obey. Thus, specifying the algebra $A$ is sufficient to obtain a consistent system of correlators. The assignment of a (suitably normalized) correlator $C(\Sigma)$ to $\Sigma$ actually depends only on the Morita class of the symmetric special Frobenius algebra $A$. Conversely, any consistent set of correlators can be obtained this way [FjFRS2].

Topological defects admit a number of interesting operations. In particular, they can be fused - on the algebraic side this corresponds to the tensor product $B \otimes_{A} B^{\prime}$ of bimodules. The bimodule morphisms $\operatorname{Hom}_{A \mid A}\left(B \otimes_{A} B^{\prime}, B^{\prime \prime}\right)$ appear as labels of vertices of defect lines. Defect lines can also be fused to boundaries; depending on the relative situation of the defect line and the boundary, this is given on the algebraic side by the tensor product $B \otimes_{A} N$ of a bimodule with a left module, or by the tensor product $N \otimes_{A} B$ with a right module, respectively.

In the following table we collect some pertinent aspects of the construction and exhibit the geometric structures on the sigma model target space $M$ that correspond to them.

\begin{tabular}{l|l|l}
\multicolumn{1}{c|}{ geometric situation } & algebraic structure in the category $\mathcal{C}$ & \multicolumn{1}{|c}{ geometric structure on $M$} \\
\hline$\Sigma$ closed oriented & symm. special Frobenius algebra $A$ & bundle gerbe $\mathcal{G}$ with connection \\
$\Sigma$ unoriented & Jandl structure $\sigma: A^{\text {opp } \rightarrow A}$ & Jandl gerbe \\
boundary condition & $A$-module & $\mathcal{G}$-D-brane \\
topological defect line & $A$-bimodule & $\mathcal{G}$-bi-brane
\end{tabular}

Jandl gerbes, D-branes and bi-branes will be presented in Sections 5, 6, and 7, respectively.

\section{Jandl gerbes: Holonomy for unoriented surfaces}

We have defined trivial bundle gerbes with connection as 2-forms because 2-forms can be integrated over oriented surfaces. Closing the 2-category of trivial bundle gerbes under descent has lead us to bundle gerbes. Jandl gerbes are bundle gerbes with additional structure, whose holonomy is defined for closed surfaces without orientation, even for unorientable surfaces [SSW]. In particular, Jandl gerbes provide Wess-Zumino terms for unoriented surfaces. Comparing the geometric data with the representation theoretic ones from Section 4 , bundle gerbes with connection correspond to Frobenius algebras, while Jandl gerbes correspond to Jandl algebras.

The appropriate quantity that has to replace 2-forms in order to make integrals over an unoriented surface well-defined is a 2-density. Every surface $\Sigma$ has an oriented double covering pr: $\hat{\Sigma} \rightarrow \Sigma$ that comes with an orientation-reversing involution $\sigma: \hat{\Sigma} \rightarrow \hat{\Sigma}$ which exchanges the two sheets and preserves the fibres. A 2 -density on $\Sigma$ is a 2 -form $\omega \in \Omega^{2}(\hat{\Sigma})$ such that

$$
\sigma^{*} \omega=-\omega
$$

Every ordinary 2-form $\rho$ on $\Sigma$ defines a particular 2-density by $\omega_{\rho}:=\operatorname{pr}^{*} \rho$. 
A 2-density on $\Sigma$ can indeed be integrated without requiring $\Sigma$ to be oriented. One chooses a dual triangulation $\Gamma$ of $\Sigma$ and, for each face $f$ of $\Gamma$, one of its two preimages under pr: $\hat{\Sigma} \rightarrow \Sigma$, denoted $f_{\text {or }}$. Then one sets

$$
\int_{\Sigma} \omega:=\sum_{f} \int_{f_{\mathrm{or}}} \omega
$$

Owing to the equality (5.1) the so defined integral does not depend on the choice of the preimages $f_{\text {or }}$ nor on the choice of triangulation $\Gamma$. If $\Sigma$ can be endowed with an orientation, the preimages $f_{\text {or }}$ can be chosen in such a way that $\left.\mathrm{pr}\right|_{f_{\text {or }}}: f_{\text {or }} \rightarrow f$ is orientation-preserving. Then the integral of a 2-density $\omega_{\rho}$ coincides with the ordinary integral of the 2-form $\rho$.

Next we want to set up a 2-category whose objects are related to 2-densities. To this end we use the 2-category of trivial bundle gerbes introduced in Section 3.2. Thus, one datum specifying an object is a 2-form $\omega \in \Omega^{2}(\hat{\Sigma})$. In the context of 2-categories, demanding strict equality as in (5.1) is unnatural. Instead, we replace equality by a 1-morphism

$$
\eta: \quad \sigma^{*} \omega \rightarrow-\omega
$$

i.e. a 1-form $\eta \in \Omega^{1}(\hat{\Sigma})$ such that $\sigma^{*} \omega=-\omega+\mathrm{d} \eta$. As we shall see in a moment, we must impose equivariance of the 1-morphism up to some 2-morphism, i.e. we need in addition a 2-isomorphism

$$
\phi: \quad \sigma^{*} \eta \Longrightarrow \eta
$$

in other words a smooth function $\phi: M \rightarrow \mathrm{U}(1)$ such that $\eta=\sigma^{*} \eta-\mathrm{i} \operatorname{dlog} \phi$. This 2-isomorphism, in turn, must satisfy the equivariance relation

$$
\sigma^{*} \phi=\phi^{-1} \text {. }
$$

Thus the objects of the 2-category are triples $(\omega, \eta, \phi)$. Let us verify that they still lead to a well-defined notion of holonomy. We choose again a dual triangulation $\Gamma$ of $\Sigma$ as well as a preimage $f_{\text {or }}$ for each of its faces. The expression (5.2) is now no longer independent of these choices, because every change creates a boundary term in the integrals of the 1-form $\eta$. To resolve this problem, we involve orientation-reserving edges: these are edges in $\Gamma$ whose adjacent faces have been lifted to opposite sheets. Since $\Gamma$ is a dual triangulation, its orientation-reversing edges form a disjoint union of piecewise smooth circles $c \subset \Sigma$. For each of these circles, we choose again a preimage $c_{\text {or }}$. It may not be possible to choose $c_{\text {or }}$ to be closed, in which case there exists a point $p^{c} \in \Sigma$ which has two preimages in $c_{\text {or }}$. We choose again one of these preimages, denoted $p_{\text {or }}^{c}$. Then

$$
\operatorname{Hol}_{\omega, \eta, \phi}:=\exp \left(2 \pi \mathrm{i}\left(\sum_{f} \int_{f_{\mathrm{or}}} \omega+\sum_{c} \int_{c_{\mathrm{or}}} \eta\right)\right) \prod_{c} \phi\left(p_{\mathrm{or}}^{c}\right)
$$

is independent of the choice of the lifts $f_{\text {or }}, c_{\text {or }}$ and $p_{\text {or }}$, and is independent of the choice of the triangulation.

More generally, let $\mathcal{M a n}_{+}$be the category of smooth manifolds with involution, whose morphisms are equivariant smooth maps. (The involution is not required to act freely.) In a first step, we want to define a presheaf

$$
\mathcal{J}_{\text {antriv }}^{\nabla}: \operatorname{Man}_{+}^{\text {opp }} \rightarrow \mathcal{C} \text { at }
$$


of trivial Jandl gerbes. For $(M, k)$ a smooth manifold with involution $k: M \rightarrow M$, a trivial Jandl gerbe involves as a first datum a trivial bundle gerbe $\mathcal{I}_{\omega}$, but as explained in Section 1 we replace the 1-morphism $\eta$ from (5.3) by a line bundle $L$ over $M$ with connection of curvature

$$
\operatorname{curv}(L)=-\omega-k^{*} \omega,
$$

and we replace the 2-isomorphism $\phi$ from (5.4) by an isomorphism $\phi: k^{*} L \rightarrow L$ of line bundles with connection, still subject to the condition (5.5). Notice that the pair $(L, \phi)$ is nothing but a $k$-equivariant line bundle with connection over $M$. After this step, we still have the holonomy (5.6), which now looks like

$$
\operatorname{Hol}_{\mathcal{I}_{\omega}, L, \phi}=\exp \left(2 \pi \mathrm{i} \sum_{f} \int_{f_{\mathrm{or}}} \omega\right) \prod_{c} \operatorname{Hol}_{\bar{L}}(c),
$$

where we have used the fact that, since the action of $\langle k\rangle$ on $c_{\text {or }}$ is free, the $k$-equivariant line bundle $(L, \phi)$ descends to a line bundle $\bar{L}$ with connection over the quotient $c=c_{\mathrm{or}} /\langle k\rangle$. This formula is now manifestly independent of the choices of $c_{\mathrm{or}}$ and $p_{\mathrm{or}}^{c}$. Its independence under different choices of faces $f_{\text {or }}$ is due to (5.8).

Now we close the presheaf $\mathcal{J}_{\text {antriv }} \nabla(M)$ under descent to allow for non-trivial bundle gerbes. To do so, we need to introduce duals of bundle gerbes, 1-morphisms and 2-isomorphisms see Wa1; for the sake of brevity we omit these definitions here.

Definition 4. Let $M$ be a smooth manifold with involution $k: M \rightarrow M$. A Jandl gerbe is a bundle gerbe $\mathcal{G}$ over $M$ together with a 1-isomorphism $\mathcal{A}: k^{*} \mathcal{G} \rightarrow \mathcal{G}^{*}$ to the dual gerbe and a 2-isomorphism $\varphi: k^{*} \mathcal{A} \Longrightarrow \mathcal{A}^{*}$ that satisfies $k^{*} \varphi=\varphi^{*-1}$.

Jandl gerbes form a sheaf

$$
\mathcal{J a n}^{\nabla}: \operatorname{Man}_{+}^{\text {opp }} \rightarrow \mathcal{C} \text { at }
$$

The gluing axiom for this sheaf has been proved in [GSW2]. We remark that the 1-isomorphism $\mathcal{A}$ may be regarded as the counterpart of a Jandl structure $\sigma$ on the Frobenius algebra $A$ that corresponds to the bundle gerbe $\mathcal{G}$, if one accepts that the dual gerbe plays the role of the opposed algebra.

Suppose we are given a Jandl gerbe $\mathcal{J}$ over a smooth manifold $M$ with involution $k$. If $\Sigma$ is a closed surface, possibly unoriented and possibly unorientable, and

$$
\Phi: \quad(\hat{\Sigma}, \sigma) \rightarrow(M, k)
$$

is a morphism in $\mathcal{M a n}_{+}^{\text {opp }}$, we can pull back the Jandl gerbe $\mathcal{J}$ from $M$ to $\hat{\Sigma}$. As in the case of ordinary surface holonomy, it then becomes trivial as a gerbe for dimensional reasons, and we can choose an isomorphism

$$
\mathcal{T}: \quad \Phi^{*} \mathcal{J} \stackrel{\sim}{\longrightarrow}\left(\mathcal{I}_{\omega}, L, \phi\right)
$$

Then we define

$$
\operatorname{Hol}_{\mathcal{J}}(\Phi):=\operatorname{Hol}_{\mathcal{I}_{\omega}, L, \phi} .
$$

This is independent of the choice of $\mathcal{T}$, because any other choice $\mathcal{T}^{\prime}$ gives rise to an isomorphism $\mathcal{T}^{\prime} \circ \mathcal{T}^{-1}$ in $\mathcal{J}_{\operatorname{antriv}}^{\nabla}(\hat{\Sigma}, \sigma)$ under which the holonomy stays unchanged. 
We have now seen that every Jandl gerbe $\mathcal{J}$ over a smooth manifold $M$ with involution $k$ has holonomies for unoriented closed surfaces and equivariant smooth maps $\Phi: \hat{\Sigma} \rightarrow M$. We thus infer that sigma models on $M$ whose fields are such maps, are defined by Jandl gerbes $\mathcal{J}$ over $M$ rather than by ordinary bundle gerbes $\mathcal{G}$. This makes it an interesting problem to classify Jandl gerbes.

Concerning the existence of a Jandl gerbe $\mathcal{J}$ with underlying bundle gerbe $\mathcal{G}$, the 1-isomorphism $\mathcal{A}: k^{*} \mathcal{G} \rightarrow \mathcal{G}^{*}$ requires the curvature $H$ of $\mathcal{G}$ to satisfy

$$
k^{*} H=-H \text {. }
$$

Apart from this necessary condition, there is a sequence of obstruction classes [GSW2]. Reduced to the case that $M$ is 2-connected, there is one obstruction class $o(\mathcal{G}) \in H^{3}\left(\mathbb{Z}_{2}, \mathrm{U}(1)\right)$, the group cohomology of $\mathbb{Z}_{2}$ with coefficients in $\mathrm{U}(1)$, on which $\mathbb{Z}_{2}$ acts by inversion. If $o(\mathcal{G})$ vanishes, then inequivalent Jandl gerbes with the same underlying bundle gerbe $\mathcal{G}$ are parameterized by $H^{2}\left(\mathbb{Z}_{2}, \mathrm{U}(1)\right)$.

These results can be made very explicit in the case of WZW models, for which the object in $\mathcal{M a n}_{+}$is a connected compact simple Lie group $G$ equipped with an involution $k: G \rightarrow G$ acting as

$$
k: \quad g \mapsto(z g)^{-1}
$$

for a fixed 'twist element' $z \in Z(G)$. It is easy to see that the 3 -form $H_{k} \in \Omega^{3}(G)$, which is the curvature of the level- $k$ bundle gerbes $\mathcal{G}$ over $G$, satisfies the necessary condition (5.14). All obstruction classes $o(\mathcal{G})$ and all parameterizing groups have been computed in dependence of the twist element $z$ and the level $k$ GSW1. The numbers of inequivalent Jandl gerbes range from two (for simply connected $G$, per level and involution) to sixteen (for $\operatorname{PSO}(4 n)$, for every even level).

Most prominently, there are two involutions on SU(2), namely $g \longmapsto g^{-1}$ and $g \longmapsto-g^{-1}$, and for each of them two inequivalent Jandl gerbes per level. On $\mathrm{SO}(3)$ there is only a single involution, but the results of [SSW, GSW1] exhibit four inequivalent Jandl gerbes per even level. This explains very nicely why $\mathrm{SU}(2)$ and $\mathrm{SO}(3)$ have the same number of orientifolds, despite a different number of involutions. These results reproduce those of the algebraic approach (see e.g. [FRS2]); for the precise comparison, Jandl structures related by the action of the trivial line bundle with either of its two equivariant structures have to be identified.

\section{D-branes: Holonomy for surfaces with boundary}

We now introduce the geometric structure needed to define surface holonomies and WessZumino terms for surfaces with boundary. When one wants to define holonomy along a curve that is not closed, one way to make the parallel transport group-valued is to choose trivializations at the end points. To incorporate these trivializations into the background, one can choose a submanifold $\dot{\mathcal{D}} \subset M$ together with a trivialization $\left.E\right|_{\dot{\mathcal{D}}} \rightarrow \mathbf{1}_{A}$. Admissible paths $\gamma:[0,1] \rightarrow M$ are then required to start and end on this submanifold, $\gamma(0), \gamma(1) \in \dot{\mathcal{D}}$. The same strategy has proven to be successful for surfaces with boundary.

Definition 5. Let $\mathcal{G}$ be a bundle gerbe with connection over $M$. A $\mathcal{G}$-D-brane is a submanifold $\dot{\mathcal{D}} \subset M$ together with a 1-morphism

$$
\mathcal{D}:\left.\quad \mathcal{G}\right|_{\mathcal{D}} \rightarrow \mathcal{I}_{\omega}
$$


to a trivial bundle gerbe $\mathcal{I}_{\omega}$ given by a two-form $\omega$ on $\dot{\mathcal{D}}$.

The morphism $\mathcal{D}$ is called a $\mathcal{G}$-module, or twisted vector bundle. Notice that if $H$ is the curvature of $\mathcal{G}$, the 1-morphism $\mathcal{D}$ enforces the identity

$$
\left.H\right|_{\dot{\mathcal{D}}}=\mathrm{d} \omega
$$

This equality restricts the possible choices of the world volume $\dot{\mathcal{D}}$ of the $\mathcal{G}$-D-brane.

Suppose that $\Sigma$ is an oriented surface, possibly with boundary, and $\Phi: \Sigma \rightarrow M$ is a smooth map. We require that $\Phi(\partial \Sigma) \subset \dot{\mathcal{D}}$. As described in Section [3.3, we choose a trivialization $\mathcal{T}: \Phi^{*} \mathcal{G} \longrightarrow \mathcal{I}_{\rho}$. Its restriction to $\partial \Sigma$ and the $\mathcal{G}$-module $\mathcal{D}$ define a 1-morphism

$$
\left.\left.\mathcal{I}_{\rho}\right|_{\partial \Sigma} \stackrel{\left.\mathcal{T}^{-1}\right|_{\partial \Sigma}}{\longrightarrow} \Phi^{*} \mathcal{G}\right|_{\partial \Sigma}=\Phi^{*}\left(\left.\mathcal{G}\right|_{\dot{\mathcal{D}}}\right) \stackrel{\Phi^{*}(\mathcal{D})}{\longrightarrow} \Phi^{*}\left(\mathcal{I}_{\omega}\right)
$$

According to the definition (3.13), this 1-morphism is nothing but a hermitian vector bundle $E$ with connection over $\partial \Sigma$ and its curvature is $\operatorname{curv}(E)=\omega-\rho$. Then we consider

$$
\operatorname{Hol}_{\mathcal{G}, \mathcal{D}}(\Phi):=\exp \left(2 \pi \mathrm{i} \int_{\Sigma} \rho\right) \operatorname{tr}\left(\operatorname{Hol}_{E}(\partial \Sigma)\right)
$$

where the trace makes the holonomy of $E$ independent of the choice of a parameterization of $\partial \Sigma$. This expression is independent of the choice of the trivialization $\mathcal{T}:$ if $\mathcal{T}^{\prime}: \mathcal{G} \rightarrow \mathcal{I}_{\rho^{\prime}}$ is another one and $E^{\prime}$ is the corresponding vector bundle, we have the transition isomorphism $L$ from (3.27) with curvature $\rho^{\prime}-\rho$, and an isomorphism $E^{\prime} \otimes L \cong E$. It follows that

$$
\exp \left(2 \pi \mathrm{i} \int_{\Sigma} \rho\right) \operatorname{tr}\left(\operatorname{Hol}_{E}(\partial \Sigma)\right)=\exp \left(2 \pi \mathrm{i}\left(\int_{\Sigma} \rho^{\prime}-\operatorname{curv}(L)\right)\right) \operatorname{tr}\left(\operatorname{Hol}_{E^{\prime} \otimes L}(\partial \Sigma)\right),
$$

and on the right hand side the unprimed quantities cancel by Stokes' theorem.

Important results on D-branes concern in particular two large classes of models, namely free field theories and again WZW theories. The simplest example of a free field theory is the one of a compactified free boson, in which $M$ is a circle $S_{R}^{1} \cong \mathbb{R} \bmod 2 \pi R \mathbb{Z}$ of radius $R$. As is well known, there are then in particular two distinct types of D-branes: D0-branes $\mathcal{D}_{x}^{(0)}$, whose support is localized at a position $x \in S_{R}^{1}$, and D1-branes $\mathcal{D}_{\alpha}^{(1)}$, whose world volume is all of $S_{R}^{1}$ and which are characterized by a Wilson line $\alpha \in \mathbb{R} \bmod \frac{1}{2 \pi R} \mathbb{Z}$, corresponding to a flat connection on $S_{R}^{1}$.

For WZW theories, which are governed by a bundle gerbe $\mathcal{G}$ over a connected compact simple Lie group $G$, preserving the non-abelian current symmetries puts additional constraints on the admissible D-branes: their support $\dot{\mathcal{D}}$ must be a conjugacy class $\mathcal{C}_{h}$ of a group element $h \in G$. This can e.g. be seen by studying the scattering of bulk fields in the presence of the D-brane. On such conjugacy classes one finds a canonical 2-form $\omega_{h} \in \Omega^{2}\left(\mathcal{C}_{h}\right)$. Additionally, the 1-morphism $\mathcal{D}:\left.\mathcal{G}\right|_{\mathcal{C}_{h}} \rightarrow \mathcal{I}_{\omega_{h}}$ of a symmetric D-brane must satisfy a certain equivariance condition Ga2]. Interestingly, only on those conjugacy classes $\mathcal{C}_{h}$ for which

$$
h=\exp \left(2 \pi \mathrm{i} \frac{\alpha+\rho}{k+g^{\vee}}\right),
$$

with $\alpha$ an integrable highest weight, admit such 1-morphisms. Here $\rho$ denotes the Weyl vector and $g^{\vee}$ the dual Coxeter number of the Lie algebra $\mathfrak{g}$ of $G$. Thus in particular the possible world volumes of symmetric D-branes form only a discrete subset of conjugacy classes. 
We finally remark that the concepts of D-branes and Jandl gerbes can be merged [GSW2]. The resulting structures provide holonomies for unoriented surfaces with boundary, and can be used to define D-branes in WZW orientifold theories.

\section{Bi-branes: Holonomy for surfaces with defect lines}

\subsection{Gerbe bimodules and bi-branes}

In the representation theoretic approach to rational conformal field theory, boundary conditions and defect lines are described as modules and bimodules, respectively. The fact that the appropriate target space structure for describing boundary conditions, D-branes, is related to gerbe modules, raises the question of what the appropriate target space structure for defect lines should be. The following definition turns out to be appropriate.

Definition 6. Let $\mathcal{G}_{1}$ and $\mathcal{G}_{2}$ be bundle gerbes with connection over $M_{1}$ and $M_{2}$, respectively. A $\mathcal{G}_{1}-\mathcal{G}_{2}$-bi-brane is a submanifold $\dot{\mathcal{B}} \subset M_{1} \times M_{2}$ together with a $\left.\left(p_{1}^{*} \mathcal{G}_{1}\right)\right|_{\dot{\mathcal{B}}}-\left.\left(p_{2}^{*} \mathcal{G}_{2}\right)\right|_{\dot{\mathcal{B}}}$-bimodule, i.e. with a 1-morphism

$$
\mathcal{B}:\left.\left.\quad\left(p_{1}^{*} \mathcal{G}_{1}\right)\right|_{\dot{\mathcal{B}}} \rightarrow\left(p_{2}^{*} \mathcal{G}_{2}\right)\right|_{\dot{\mathcal{B}}} \otimes \mathcal{I}_{\varpi}
$$

with $\mathcal{I}_{\varpi}$ a trivial bundle gerbe given by a two-form $\varpi$ on $\dot{\mathcal{B}}$.

Similarly as in (6.2) it follows that the two-form $\varpi$ on $\dot{\mathcal{B}}$ obeys

$$
\left.p_{1}^{*} H\right|_{\dot{\mathcal{B}}}=\left.p_{2}^{*} H\right|_{\dot{\mathcal{B}}}+\mathrm{d} \varpi
$$

We call $\dot{\mathcal{B}}$ the world volume and $\varpi$ the curvature of the bimodule. With the appropriate notion of duality for bundle gerbes (see Section 1.4 of [Wa1]), a $\mathcal{G}_{1}-\mathcal{G}_{2}$-bimodule is the same as a $\left(\mathcal{G}_{1} \otimes \mathcal{G}_{2}^{*}\right)$-module. For a formulation in terms of local data, see (B.8) of [FSW].

As an illustration, consider again the free boson and WZW theories, restricting attention to the case $M_{1}=M_{2}$. For the free boson compactified on a circle $S_{R}^{1}$ of radius $R$, one finds that the world volume of a bi-brane is a submanifold $\dot{\mathcal{B}}_{x} \subset S_{R}^{1} \times S_{R}^{1}$ of the form

$$
\dot{\mathcal{B}}_{x, \alpha}:=\left\{(y, y-x) \mid y \in S_{R}^{1}\right\}
$$

with $x \in S_{R}^{1}$. The submanifold $\dot{\mathcal{B}}_{x, \alpha}$ has the topology of a circle and comes with a flat connection, i.e. with a Wilson line $\alpha$. Thus the bi-branes of a compactified free boson are naturally parameterized by a pair $(x, \alpha)$ taking values in two dual circles that describe a point on $S_{R}^{1}$ and a Wilson line.

In the WZW case, for which the target space is a compact connected simple Lie group $G$, a scattering calculation [FSW] similar to the one performed for D-branes indicates that the world volume of a (maximally symmetric) bi-brane is a biconjugacy class

$$
\dot{\mathcal{B}}_{h, h^{\prime}}:=\left\{\left(g, g^{\prime}\right) \in G \times G \mid \exists x_{1}, x_{2} \in G: g=x_{1} h x_{2}^{-1}, g^{\prime}=x_{1} h^{\prime} x_{2}^{-1}\right\} \subset G \times G
$$

of a pair $\left(h, h^{\prime}\right)$ of group elements satisfying $h\left(h^{\prime}\right)^{-1} \in \mathcal{C}_{h_{\alpha}}$ with $h_{\alpha}$ as given in (6.6). The biconjugacy classes carry two commuting $G$-actions, corresponding to the presence of two independent conserved currents in the field theory. Further, a biconjugacy class can be described as the preimage

$$
\dot{\mathcal{B}}_{h, h^{\prime}}=\tilde{\mu}^{-1}\left(\mathcal{C}_{h h^{\prime-1}}\right)=\left\{\left(g, g^{\prime}\right) \in G \times G \mid g g^{\prime-1} \in \mathcal{C}_{h h^{\prime-1}}\right\}
$$


of the conjugacy class $\mathcal{C}_{h h^{\prime-1}}$ under the map

$$
\tilde{\mu}: \quad G \times G \ni\left(g_{1}, g_{2}\right) \longmapsto g_{1} g_{2}^{-1} \in G .
$$

Finally, the relevant two-form on $\dot{\mathcal{B}}_{h, h^{\prime}}$ is

$$
\varpi_{h, h^{\prime}}:=\tilde{\mu}^{*} \omega_{h h^{\prime-1}}-\frac{k}{2}\left\langle p_{1}^{*} \theta \wedge p_{2}^{*} \theta\right\rangle .
$$

Here $k$ is the level, $\theta$ is the left-invariant Maurer-Cartan form, $p_{i}$ are the projections to the factors of $G \times G$, and $\omega_{h}$ is the canonical 2-form (see Section 6) on the conjugacy class $\mathcal{C}_{h}$. One checks that $\varpi_{h, h^{\prime}}$ is bi-invariant and satisfies (7.2).

Examples of symmetric bi-branes can be constructed from symmetric D-branes using a multiplicative structure on the bundle gerbe $\mathcal{G}$ [Wa2]. Another important class of examples are Poincaré line bundles. These describe T-dualities; an elementary relation between T-duality

and Poincaré line bundles is provided [SaS] by the equation of motion [RS] in the presence of defects.

\subsection{Holonomy and Wess-Zumino term for defects}

The notion of bi-brane allows one in particular to define holonomy also for surfaces with defect lines.

The simplest world sheet geometry involving a defect line consists of a closed oriented world sheet $\Sigma$ together with an embedded oriented circle $S \subset \Sigma$ that separates the world sheet into two components, $\Sigma=\Sigma_{1} \cup_{S} \Sigma_{2}$. Assume that the defect $S$ separates regions that support conformally invariant sigma models with target spaces $M_{1}$ and $M_{2}$, respectively, and consider maps $\phi_{i}: \Sigma_{i} \rightarrow M_{i}$ for $i \in\{1,2\}$ such that the image of

$$
\begin{aligned}
\phi_{S}: S & \longrightarrow M_{1} \times M_{2} \\
s & \mapsto\left(\phi_{1}(s), \phi_{2}(s)\right)
\end{aligned}
$$

is contained in the submanifold $\dot{\mathcal{B}}$ of $M_{1} \times M_{2}$. The orientation of $\Sigma_{i}$ is the one inherited from the orientation of $\Sigma$, and without loss of generality we take $\partial \Sigma_{1}=S$ and $\partial \Sigma_{2}=-S$.

We wish to find the Wess-Zumino part of the sigma model action, or rather the corresponding holonomy $\operatorname{Hol}_{\mathcal{G}_{1}, \mathcal{G}_{2}, \mathcal{B}}$, that corresponds to having bundle gerbes $\mathcal{G}_{1}$ and $\mathcal{G}_{2}$ over $M_{1}$ and $M_{2}$ and a $\mathcal{G}_{1}-\mathcal{G}_{2}$-bi-brane $\mathcal{B}$. The pullback of the bimodule (7.1) along the map $\phi_{S}: S \rightarrow \dot{\mathcal{B}}$ gives a $\left.\left.\left(\phi_{1}^{*} \mathcal{G}_{1}\right)\right|_{S^{-}}\left(\phi_{2}^{*} \mathcal{G}_{2}\right)\right|_{S^{-}}$bimodule

$$
\phi_{S}^{*} \mathcal{B}:\left.\left.\quad\left(\phi_{1}^{*} \mathcal{G}_{1}\right)\right|_{S} \rightarrow\left(\phi_{2}^{*} \mathcal{G}_{2}\right)\right|_{S} \otimes \mathcal{I}_{\phi_{S}^{*} \varpi}
$$

The pullback bundle gerbes $\phi_{i}^{*} \mathcal{G}_{i}$ over $\Sigma_{i}$ are trivializable for dimensional reasons, and a choice $\mathcal{T}_{i}: \phi_{i}^{*} \mathcal{G}_{i} \rightarrow \mathcal{I}_{\rho}$ of trivializations for two-forms $\rho_{i}$ on $\Sigma_{i}$ produces a vector bundle $E$ over $S$. We then define

$$
\operatorname{Hol}_{\mathcal{G}_{1}, \mathcal{G}_{2}, \mathcal{B}}(\Sigma, S):=\exp \left(2 \pi \mathrm{i} \int_{\Sigma_{1}} \rho_{1}\right) \exp \left(2 \pi \mathrm{i} \int_{\Sigma_{2}} \rho_{2}\right) \operatorname{tr}\left(\operatorname{Hol}_{E}(S)\right) \in \mathbb{C}
$$

to be the holonomy in the presence of the bi-brane $\mathcal{B}$. As shown in Appendix B.3 of [FSW], for similar reasons as in the case of D-branes the number $\operatorname{Hol}_{\mathcal{G}_{1}, \mathcal{G}_{2}, \mathcal{B}}(\Sigma, S)$ is independent of the choice of the trivializations $\mathcal{T}_{1}$ and $\mathcal{T}_{2}$. 


\subsection{Fusion of defects}

In the field theory context of section 4 there are natural notions of the fusion of a defect (an $A$ bimodule) with a boundary condition (a left $A$-module), yielding another boundary condition, and of the fusion of two defects, yielding another defect. Both of these are provided by the tensor product over the relevant Frobenius algebra $A$. These representation theoretic notions of fusion have a counterpart on the geometric side as well.

Consider first the fusion of a defect with a boundary condition. We allow for the general situation of a defect described by an $M_{1}-M_{2}$-bi-brane with different target spaces $M_{1}$ and $M_{2}$. Thus take an $M_{1}-M_{2}$-bi-brane with world volume $\dot{\mathcal{B}} \subseteq M_{1} \times M_{2}$ and an $M_{2}$-D-brane with world volume $\dot{\mathcal{D}} \subseteq M_{2}$. The action of correspondences on sheaves suggests the following ansatz for the world volume of the fusion product:

$$
(\mathcal{B} \star \mathcal{D})^{\cdot}:=p_{1}\left(\dot{\mathcal{B}} \cap p_{2}^{-1}(\dot{\mathcal{D}})\right)
$$

with $p_{i}$ the projection $M_{1} \times M_{2} \rightarrow M_{i}$. The corresponding ansatz for the fusion of an $M_{1^{-}} M_{2^{-}}$ bi-brane $\mathcal{B}$ of world volume $\dot{\mathcal{B}}$ with an $M_{2}-M_{3}$-bi-brane $\mathcal{B}^{\prime}$ of world volume $\dot{\mathcal{B}}^{\prime}$ uses projections $p_{i j}$ from $M_{1} \times M_{2} \times M_{3}$ to $M_{i} \times M_{j}$ :

$$
\left(\mathcal{B} \star \mathcal{B}^{\prime}\right)^{\cdot}:=p_{13}\left(p_{12}^{-1}(\dot{\mathcal{B}}) \cap p_{23}^{-1}\left(\dot{\mathcal{B}}^{\prime}\right)\right) .
$$

In general one obtains this way only subsets, rather than submanifolds, of $M_{1}$ and $M_{1} \times M_{3}$, respectively. On a heuristic level one would, however, expect that owing to quantization of the branes a finite superposition of branes is selected, which should then reproduce the results obtained in the field theory setting.

We illustrate this again with the two classes of models already considered, i.e. free bosons and WZW theories, again restricting attention to the case $M_{1}=M_{2}$. First, for the theory of a compactified free boson, the D-brane is of one of the types $\mathcal{D}_{x}^{(0)}$ or $\mathcal{D}_{\alpha}^{(1)}$ (see Section 6) and the bi-brane world volume is of the form $\dot{\mathcal{B}}_{x, \alpha}$ given in $(7.3)$. For D-branes of type $\mathcal{D}_{x}^{(0)}$ the prescription (7.11) thus yields

$$
\mathcal{B}_{(x, \alpha)} \star \mathcal{D}_{y}^{(0)}=\mathcal{D}_{x+y}^{(0)}
$$

For the fusion of a bi-brane $\mathcal{B}_{(x, \alpha)}$ and a D1-brane $\mathcal{D}_{\beta}^{(1)}$, one must take the flat line bundle on the bi-brane into account. We first pull back the line bundle on $\dot{\mathcal{D}}_{\beta}^{(1)}$ along $p_{2}$ to a line bundle on $S_{R}^{1} \times S_{R}^{1}$, then restrict it to $\dot{\mathcal{B}}_{(x, \alpha)}$, and finally tensor this restriction with the line bundle on $\dot{\mathcal{B}}_{(x, \alpha)}$ described by the Wilson line $\alpha$. This results in a line bundle with Wilson line $\alpha+\beta$ on the bi-brane world volume, which in turn can be pushed down along $p_{1}$ to a line bundle on $S_{R}^{1}$, so that

$$
\mathcal{B}_{(x, \alpha)} \star \mathcal{D}_{\beta}^{(1)}=\mathcal{D}_{\alpha+\beta}^{(1)}
$$

In short, the fusion with a defect $\mathcal{B}_{(x, \alpha)}$ acts on D0-branes as a translation by $x$ in position space, and on D1-branes as a translation by $\alpha$ in the space of Wilson lines. Similarly, the prescription (7.12) leads to

$$
\mathcal{B}_{(x, \alpha)} \star \mathcal{B}_{\left(x^{\prime}, \alpha^{\prime}\right)}=\mathcal{B}_{\left(x+x^{\prime}, \alpha+\alpha^{\prime}\right)}
$$

for the fusion of two bi-branes $\mathcal{B}_{(x, \alpha)}$ and $\mathcal{B}_{\left(x^{\prime}, \alpha^{\prime}\right)}$, i.e. both the position and the Wilson line variable of the bi-branes add up. 
For WZW theories, besides the quantization of the positions of the branes another new phenomenon is that multiplicities other than zero or one appear in the field theory approach. In that context they arise from the decomposition $B_{\alpha} \otimes_{A} B_{\beta}=\bigoplus_{\gamma} \mathcal{N}_{\alpha \beta}^{\gamma} B_{\gamma}$ of a tensor product of simple $A$-bimodules into a finite direct sum of simple $A$-bimodules, and analogously for the case of mixed fusion (in rational CFT, both the category of $A$-modules and the category of $A$ bimodules are semisimple). Moreover, for simply connected groups, the multiplicities appearing in both types of fusion are in fact the same as the chiral fusion multiplicities which are given by the Verlinde formula.

By analogy with the field theory situation we expect fusion rules

$$
\mathcal{B}_{\alpha} \star \mathcal{B}_{\beta}=\sum_{\gamma} \mathcal{N}_{\alpha \beta}{ }^{\gamma} \mathcal{B}_{\gamma}
$$

of bi-branes, and analogously for mixed fusion of bi-branes and D-branes. In the particular case of WZW theories on simply connected Lie groups one can in addition invoke the duality $\alpha \longmapsto \alpha^{\vee}$ which in that case exists on the sets of branes as well as defects that preserve all current symmetries, so as to work instead with fusion coefficients of type $\mathcal{N}_{\alpha \beta \gamma}=\mathcal{N}_{\alpha \beta} \gamma^{\vee}$. Then for the case of two D-branes $\mathcal{D}_{\alpha}$ and $\mathcal{D}_{\gamma}$ with world volumes given by conjugacy classes $\mathcal{C}_{h_{\alpha}}$ and $\mathcal{C}_{h_{\gamma}}$ of $G$, as well as a bi-brane $\mathcal{B}_{\beta}$ whose world volume is the biconjugacy class $\tilde{\mu}^{-1}\left(\mathcal{C}_{h_{\beta}}\right)$, one is lead to consider the subset

$$
\Pi_{\alpha \beta \gamma}:=p_{1}^{-1}\left(\mathcal{C}_{\alpha}\right) \cap \tilde{\mu}^{-1}\left(\mathcal{C}_{\beta}\right) \cap p_{2}^{-1}\left(\mathcal{C}_{\gamma}\right)=\left\{\left(g, g^{\prime}\right) \in G \times G \mid g \in \mathcal{C}_{\alpha}, g^{\prime} \in \mathcal{C}_{\gamma}, g g^{\prime-1} \in \mathcal{C}_{\beta}\right\}
$$

of $G \times G$. Combining the adjoint action on $g$ and on $g^{\prime}$ gives a natural $G$-action on $\Pi_{\alpha \beta \gamma}$. And since both D-branes and the bi-brane are equipped with two-forms $\omega_{\alpha}, \omega_{\gamma}$ and $\varpi_{\beta}, \Pi_{\alpha \beta \gamma}$ comes with a natural two-form as well, namely with

$$
\omega_{\alpha \beta \gamma}:=\left.p_{1}^{*} \omega_{\alpha}\right|_{\Pi_{\alpha \beta \gamma}}+\left.p_{2}^{*} \omega_{\gamma}\right|_{\Pi_{\alpha \beta \gamma}}+\left.\varpi_{\beta}\right|_{\Pi_{\alpha \beta \gamma}} .
$$

By comparison with the field theory approach, this result should be linked to the fusion rules of the chiral WZW theory and thereby provide a physically motivated realization of the Verlinde algebra. To see how such a relation can exist, notice that fusion rules are dimensions of spaces of conformal blocks and as such can be obtained by geometric quantization from suitable moduli spaces of flat connections which arise in the quantization of Chern-Simons theories (see e.g. [ADW]). The moduli space relevant to us is the one for the three-punctured sphere $S_{(3)}^{2}$, for which the monodromy of the flat connection around the punctures takes values in conjugacy classes $\mathcal{C}_{\alpha}, \mathcal{C}_{\beta}$ and $\mathcal{C}_{\gamma}$, respectively. The relations in the fundamental group of $S_{(3)}^{2}$ imply the condition $g_{\alpha} g_{\beta} g_{\gamma}=1$ on the monodromies $g_{\alpha} \in \mathcal{C}_{\alpha}, g_{\beta} \in \mathcal{C}_{\beta}$ and $g_{\gamma} \in \mathcal{C}_{\gamma}$. Since monodromies are defined only up to simultaneous conjugation, the moduli space that matters in classical Chern-Simons theory is isomorphic to the quotient $\Pi_{\alpha \beta \gamma} / G$.

It turns out that the range of bi-branes appearing in the fusion product is correctly bounded already before geometric quantization. Indeed, the relevant product of conjugacy classes is

$$
\mathcal{C}_{h} * \mathcal{C}_{h^{\prime}}:=\left\{g g^{\prime} \mid g \in \mathcal{C}_{h}, g^{\prime} \in \mathcal{C}_{h^{\prime}}\right\}
$$

and for the case of $G=\mathrm{SU}(2)$ it is easy to see that this yields the correct upper and lower bounds for the SU(2) fusion rules [JW, FSW]. A full understanding of fusion can, however, only be expected after applying geometric quantization to the so obtained moduli space: this 
space must be endowed with a two-form, which is interpreted as the curvature of a line bundle, and the holomorphic sections of this bundle are what results from geometric quantization. In view of this need for quantization it is a highly non-trivial observation that the two-form (7.18) furnished by the two branes and the bi-brane is exactly the same as the one that arises from classical Chern-Simons theory.

In terms of defect lines, the decomposition (7.16) of the fusion product of bi-branes corresponds to the presence of a defect junction, which constitutes a particular type of defect field. A sigma model description for world sheets with such embedded defect junctions has been proposed in [RS].

We have demonstrated how structural analogies between the geometry of bundle gerbes and the representation theoretic approach to rational conformal field theory lead to interesting geometric structure, including a physically motivated realization of the Verlinde algebra. The precise form of the latter and its relation with the realization of the Verlinde algebra in the context of supersymmetric conformal field theory [FHT] remain to be understood. But in any case the parallelism between classical actions and full quantum theory exhibited above remains intriguing and raises the hope that some of the structural aspects discussed in this contribution are generic features of quantum field theories.

\section{References}

[Al] O. Alvarez, Topological quantization and cohomology, Commun. Math. Phys. 100 (1985) 279

[ADW] S. Axelrod, S. Della Pietra, and E. Witten, Geometric quantization of Chern-Simons theory, J. Diff. Geom. 33 (1991) 787

[BK] B. Bakalov and A.A. Kirillov, Lectures on Tensor Categories and Modular Functors (American Mathematical Society, Providence 2001)

[FFFS] G. Felder, J. Fröhlich, J. Fuchs, and C. Schweigert, Correlation functions and boundary conditions in RCFT and three-dimensional topology, Compos. Math. 131 (2002) 189 [hep-th/9912239]

[FjFRS1] J. Fjelstad, J. Fuchs, I. Runkel, and C. Schweigert, TFT construction of RCFT correlators V: Proof of modular invariance and factorisation, Theory and Appl. Cat. 16 (2006) 342 [hep-th/0503194]

[FjFRS2] J. Fjelstad, J. Fuchs, I. Runkel, and C. Schweigert, Uniqueness of open/closed rational CFT with given algebra of open states, Adv. Theor. Math. Phys. 12 (2008) 1281 [hep-th/0612306]

[FHT] D.S. Freed, M.J. Hopkins, and C. Teleman, Loop groups and twisted K-theory I, preprint math.AT/0711.1906

[FrB] E. Frenkel and D. Ben-Zvi, Vertex Algebras and Algebraic Curves, second edition (American Mathematical Society, Providence 2004)

[FRS1] J. Fuchs, I. Runkel, and C. Schweigert, TFT construction of RCFT correlators I: Partition functions, Nucl. Phys. B 646 (2002) 353 [hep-th/0204148]

[FRS2] J. Fuchs, I. Runkel, and C. Schweigert, TFT construction of RCFT correlators II: Unoriented world sheets, Nucl. Phys. B 678 (2004) 511 [hep-th/0306164] 
[FRS3] J. Fuchs, I. Runkel, and C. Schweigert, TFT construction of RCFT correlators IV: Structure constants and correlation functions, Nucl. Phys. B 715 (2005) 539 [hep-th/0412290]

[FRS4] J. Fuchs, I. Runkel and C. Schweigert, Ribbon categories and (unoriented) CFT: Frobenius algebras, automorphisms, reversions, Contemp. Math. 431 (2007) 203 [math.CT/0511590]

[FSW] J. Fuchs, C. Schweigert, and K. Waldorf, Bi-branes: Target space geometry for world sheet topological defects, J. Geom. and Phys. 58 (2008) 576 [hep-th/0703145]

[Ga1] K. Gawȩdzki, Topological actions in two-dimensional quantum field theories, in: Nonperturbative Quantum Field Theory, G. 't Hooft, A. Jaffe, G. Mack, P.K. Mitter, and R. Stora, eds. (Plenum, New York 1988), p. 101

[Ga2] K. Gawędzki, Abelian and non-Abelian branes in WZW models and gerbes, Commun. Math. Phys. 258 (2005) 23 [hep-th/0406072]

[GR] K. Gawędzki and N. Reis, WZW branes and gerbes, Rev. Math. Phys. 14 (2002) 1281 [hep-th/0205233]

[GSW1] K. Gawędzki, R. R. Suszek and K. Waldorf, WZW orientifolds and finite group cohomology, Commun. Math. Phys. 284 (2008) 1 [hep-th/0701071]

[GSW2] K. Gawȩdzki, R. R. Suszek and K. Waldorf, Bundle gerbes for orientifold sigma models, preprint 0809.5125

[Hu] Y.-Z. Huang, Vertex operator algebras, the Verlinde conjecture and modular tensor categories, Proc. Natl. Acad. Sci. USA 102 (2005) 5352 [math.QA/0412261]

[JW] L.C. Jeffrey and J. Weitsman, Bohr-Sommerfeld orbits in the moduli space of flat connections and the Verlinde dimension formula, Commun. Math. Phys. 150 (1992) 593

[KRT] C. Kassel, M. Rosso, and V. Turaev, Quantum Groups and Knot Invariants (Soc. Math. de France, Paris 1997)

[RS] I. Runkel and R.R. Suszek, Gerbe-holonomy for surfaces with defect networks, preprint 0808.1419

[SaS] G. Sarkissian and C. Schweigert, Some remarks on defects and T-duality, preprint 0810.3159

[SSW] U. Schreiber, C. Schweigert, and K. Waldorf, Unoriented WZW models and holonomy of bundle gerbes, Commun. Math. Phys. 274 (2007) 31 [hep-th/0512283]

[SFR] C. Schweigert, J. Fuchs, and I. Runkel, Categorification and correlation functions in conformal field theory, in: Proceedings of the International Congress of Mathematicians 2006, M. Sanz-Solé, J. Soria, J.L. Varona, and J. Verdera, eds. (European Mathematical Society, Zürich 2006), p. 443 [math.CT/0602079]

[Tu] V.G. Turaev, Quantum Invariants of Knots and 3-Manifolds (de Gruyter, New York 1994)

[Wa1] K. Waldorf, More morphisms between bundle gerbes, Theory and Appl. Cat. 17 (2007) 240 [math.CT/0702652]

[Wa2] K. Waldorf, Multiplicative bundle gerbes with connection, preprint 0804.4835 